\title{
Malignant change and multiple metastases in Ollier's disease
}

\author{
W. K. COWAN \\ From the Department of Pathology, David Lewis Northern Hospital, Liverpool
}

SYNOPSIS An example of chondrosarcoma developing in a patient with Ollier's disease is described. Necropsy showed an unusual pattern of multiple metastases including several within the cranial cavity.

Although the development of chondrosarcoma in Ollier's disease is a well recognized complication, the opportunity of examining cases of Ollier's disease at necropsy does not often occur. The case reported here is remarkable for the widespread pattern of the metastatic lesions, especially those that occurred within the skull and led to the patient's death.

\section{CASE REPORT}

The patient was a 30-year-old warehouse porter suffering from enchondromatosis; the lesions were distributed in a unilateral manner and involved the left humerus, radius, ulnar, third and fourth metacarpals and phalanges, the left scapula and ribs, the left femur, the left tibia, and the phalanges of the left foot. As a child he had sustained fractures, probably through areas of cartilaginous defect, producing deformities of both the left arm and leg. In 1947 , the leg was lengthened $6.5 \mathrm{~cm}$. by operation on the tibia. In 1951 he first noticed a dark blue, enlarging lesion on the prepuce; this was excised six months later at another hospital and, because of a diagnosis of malignant melanoma, a bilateral block dissection of inguinal nodes was performed. No tumour was found in any node. When the case came to necropsy, as is described below, multiple metastatic nodules were found; this called for a reappraisal of the original biopsy material, and I am grateful to the pathologist who immediately sent a section. It is clear that this should be regarded as a neural melanonaevus, probably benign, a diagnosis which has been confirmed by three experts. In 1960 the enchondroma at the lower end of the left tibia became painful and a little tender. Biopsy showed areas of moderately well differentiated cartilage and others of spindle cells lying in a myxochondroid matrix, features of an aggressive quality.

Six months later he was re-admitted after a fracture of the left femur occurring at the site of a pre-existing enchondroma. While this pathological fracture was apparently healing satisfactorily, he sustained another in the left tibia at the site of the previous lesion. Earlier radioReceived for publication 26 January 1965. logical suspicions of malignant change in this tibial lesion were now confirmed by radiographs showing unequivocal evidence of malignancy (Fig. 1). Biopsy showed changes typical of chondrosarcoma (Fig. 2) and a midthigh amputation was carried out.

After discharge, he was lost to follow-up from October 1961 until February 1964, when he was re-admitted with headache and photophobia. Flaccid quadriplegia developed rapidly and he died within 24 hours of admission.

\section{NECROPSY}

There was a bowing deformity of the left forearm and rounded swellings of the third and fourth fingers were present. A subcutaneous tumour over the right shoulder measured $2 \mathrm{~cm}$. in diameter and was raised approximately $1 \mathrm{~cm}$. The brain showed flattening of the gyri. In the posterior cranial fossa, a metastasis, $1.5 \mathrm{~cm}$. in diameter and covered by blood clot, was situated just left of the midline beneath and attached to the tentorium cerebelli. This tumour had also penetrated the subarachnoid space on the inferior aspect of the cerebellum. Small cartilaginous metastatic deposits were found in the leptomeninges on the superior surface of the right occipital lobe and in the cortex of the left occipital lobe. The lungs presented a metastasis, $5 \mathrm{~cm}$. in diameter in the depth of the left upper lobe, and three small metastases on the diaphragmatic surface of the left lower lobe. The left kidney contained several metastases, each approximately $3 \mathrm{~mm}$. in diameter. Two metastases were found in the spleen, each approximately $1 \mathrm{~cm}$. in diameter. The gastrointestinal tract was normal except for the jejunum, in the wall of which there were several small metastases. Multiple metastases were also observed in the mesentery. The subcutaneous lesion on the right shoulder was firm and pearly white in colour and appeared to be metastatic in nature. No tumour 


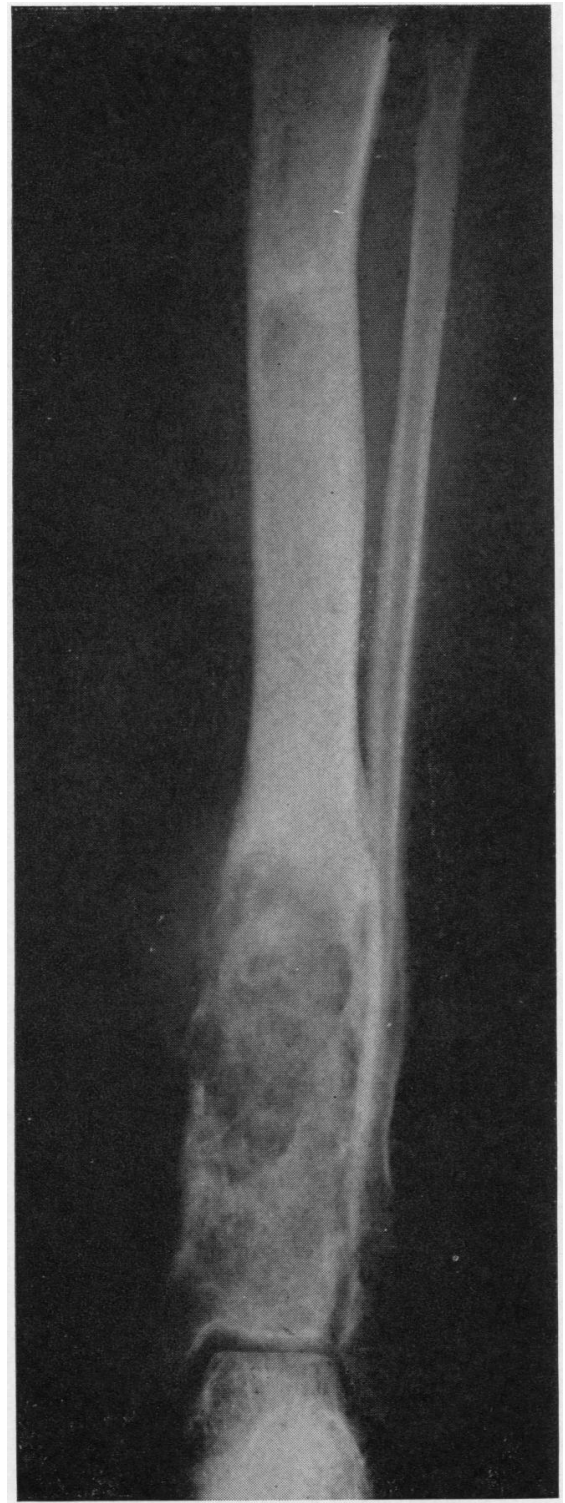

FIG. 1. Radiograph of left lower leg, showing chondrosarcoma of the tibia with destruction of the cortex and soft tissue swelling. An enchondroma is seen higher up the shaft.

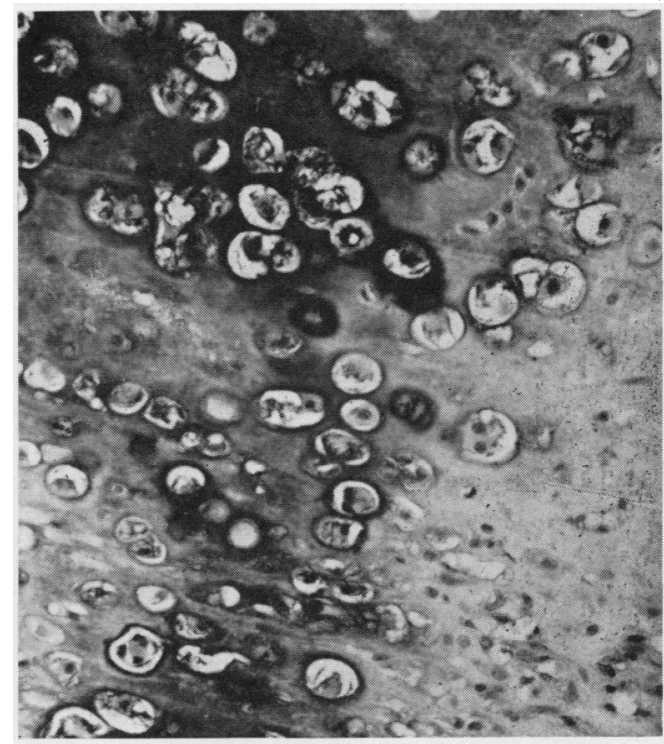

FIG. 2. Chondrosarcoma of the lower tibia showing bizarre and double nuclei. Haematoxylin and eosin $\times 180$.

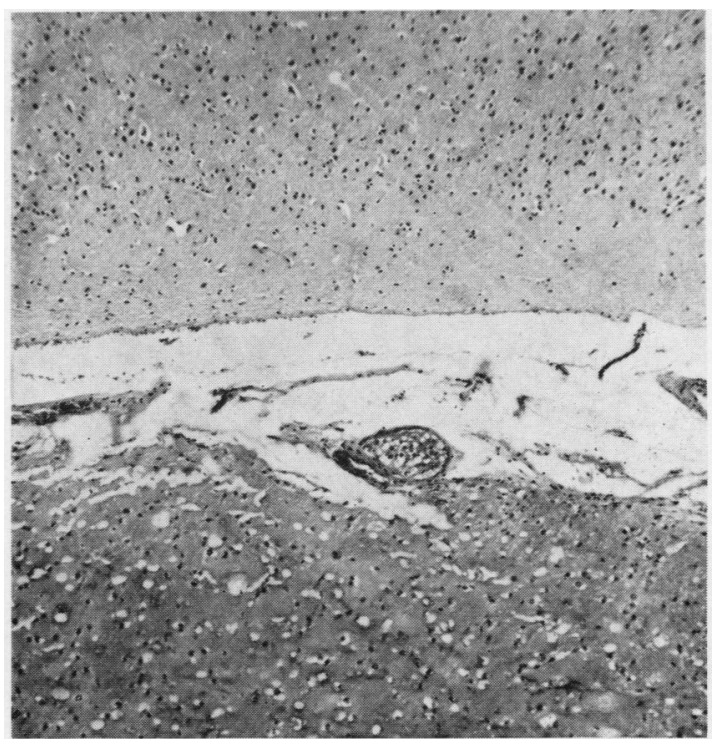

FIG. 3. Left occipital lobe, showing metastatic chondrosarcoma. Haematoxylin and eosin $\times 25$. 


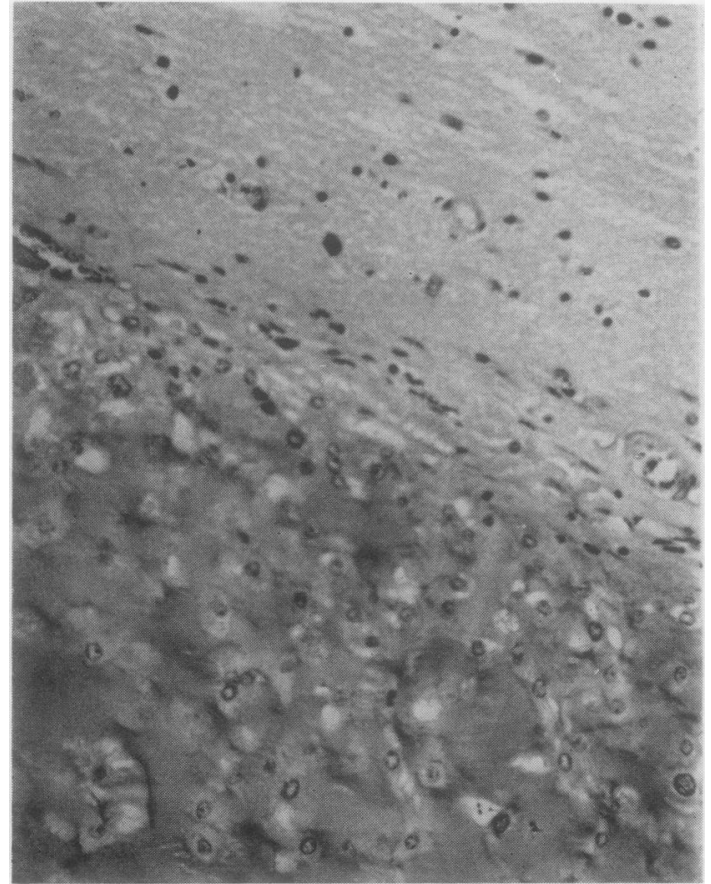

FIG. 4. Detail of cerebral metastasis shown in Fig. 3. Haematoxylin and eosin $\times 180$.

was found in the iliac veins or inferior vena cava, or in any lymph node. There was no evidence of malignant melanoma.

Microscopic examination of the lesions in the brain (Figs. 3, 4), left kidney, spleen, and jejunum as well as those of the left lung and skin, confirmed that they were metastatic chondrosarcoma.

\section{DISCUSSION}

Ollier's disease or multiple enchondromatosis is a dyschondroplasia of endochondrial ossification that is often unilateral in distribution, and the patient originally described by Ollier (1900), like the patient under discussion, presented strictly unilateral lesions.

Chondrosarcoma occurring as a complication is not rare, and several of the reported series of chondrosarcoma contain at least one example of Ollier's disease. Thus Lichtenstein and Jaffe (1943) describe two examples among their 15 patients with chondrosarcoma, Coley and Higinbotham (1954) found three cases in their series of 52 secondary chondrosarcomas, and Eyre-Brook (1961) one example in 33 patients. In the series of 40 patients with chondrosarcoma reported by O'Neal and Ackerman (1952), however, there were no cases of Ollier's disease.

From well-documented reports such as these, some appreciation of the incidence of Ollier's disease in chondrosarcoma can be obtained; however, the risk to an individual with Ollier's disease of developing chondrosarcoma is difficult to determine.

Hunter and Wiles (1935), in reviewing 40 examples of Ollier's disease, considered that the incidence of malignant transformation could not be determined; ${ }^{\circ}$ Jaffe (1958), although agreeing that the incidence was $\vec{\circ}$

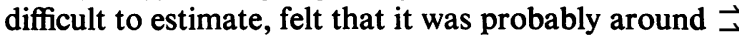
$50 \%$ and possibly higher. But any such estimate ${ }_{\stackrel{\omega}{~}}$ clearly depends on the criteria of malignancy that the pathologist lays down. Early malignant transformation in an enchondroma is associated with subtle $\infty$ microscopic changes that may or may not be accepted as valid criteria of malignancy by different $\mathrm{G}$ authorities. Certainly the interpretation of the $\circ$ histological features of Ollier's disease, where the nuclei tend to be pleomorphic, large, and occasion- $क$ ally double, is especially difficult.

The finding in the present case of cartilaginous deposits within the cranial cavity poses the question of whether they were, in fact, metastatic. Chondromatous meningioma, although a rare lesion, must $\vec{b}$ be a consideration, but it is necessary to postulate $\mathcal{O}^{\circ}$ both multiple intracranial tumours and distant metastases. Furthermore, the development of chondrosarcoma of the tibia is not accounted for by this theory and there is no known association between chondromatous meningioma and Ollier's disease. Russell and Rubinstein (1959) have observed a patient with Ollier's disease who had a myxochondrosarcoma of the base of the skull complicated by terminal subdural haemorrhage; but in the case described here, the intracranial lesions were not in continuity with the bones of the skull, and although chondromas have been reported as arising from dura mater or falx without obvious connexions to bone (Forsythe, Baker, Dockerty, and Camp, 1947), again there is no known association between this condition and Ollier's disease. Finally, mesenchymal chondrosarcoma, a tumour that may metastasize widely, has been reported as arising from the meninges (Dahlin and Henderson, 1962; Dowling, 1964), but the histological appearances of the tumour of in the present case, and in particular the lack of an $N$ anaplastic stroma are not compatible with such a diagnosis. Hence, although such lesions have not previously been described, the intracranial tumours in the present instance must be regarded as metastatic in nature.

Malignant change in Ollier's disease followed by widespread metastatic disease is exceptionally rare. Indeed extensive metastatic spread of chondrosarcoma is in general uncommon, and the usual behaviour is local invasion, local recurrence, or 
metastasis restricted to lungs, the peculiar predilection for the lungs being so marked as to encourage removal of pulmonary metastases as a curative measure. Occasionally invasion of veins and propagation in continuity along the lumen without metastasis is a feature of these tumours (Kósa, 1929; Warren, 1931).

There is evidence to suggest that the prognosis of chondrosarcoma can be related to the degree of histological differentiation and that the welldifferentiated tumours are not likely to metastasize (O'Neal and Ackerman, 1952); in this respect the present case is not atypical. However, the reported cases of chondrosarcoma exhibiting extensive or visceral metastases have in the great majority of instances been those where the primary lesion has been near to the central axis, especially the pelvis (Pasternack, Lillie, and Jones, 1933; Morton and Mider, 1947), and widespread metastases from a lesion of the distal tibia is exceptional.
I am indebted to Mr. G. E. Thomas for permission to publish details of this case and to Dr. R. Winston Evans for advice and criticism.

\section{REFERENCES}

Coley, B. L., and Higinbotham, N. L. (1954). Ann. Surg., 139, 547.

Dahlin, D. C., and Henderson, E. D. (1962). Cancer (Philad.), 15, 410.

Dowling, E. A. (1964). J. Bone Jt Surg., 46A, 747.

Eyre-Brook, A. L. (1961). Proc, roy. Soc. Med., 54, 595.

Forsythe, R. W., Baker, G. S., Dockerty, M. B., and Camp, J. D. (1947). Proc. Mayo Clin., 22, 350.

Hunter, D., and Wiles, P. (1935). Brit. J. Surg., 22, 507.

Jaffe, H. L. (1958). Tumors and tumorous Conditions of the Bones and Joints, p. 194. Kimpton, London.

Kósa, M. (1929). Virchows Arch. Path. Anat., 272, 166.

Lichtenstein, L., and Jaffe, H. L. (1943). Amer. J. Path., 19, 553.

Morton, J. J., and Mider, G. B. (1947). Ann. Surg., 126, 895.

Ollier, L. (1900). Rev. Chir. (Paris), 21, 396.

O'Neal, L. W., and Ackerman, L. V. (1952). Cancer (Philad.), 5, 551.

Pasternack, J. G., Lillie, R. D., and Jones, R. A. (1933). Arch. Path., $15,649$.

Russell, D. S., and Rubinstein, L. J. (1959). Pathology of Tumours of the Nervous System, p. 214. Arnold, London.

Warren, S. (1931). Amer. J. Path., 7, 161. 